\title{
Effectiveness of the Healthy
} e-Elderly People Intervention (HEPI) mobile application for the reduction of physical health effects caused by smartphone usage among elderly individuals in Thailand

\author{
Wachiraporn Wilaiwan \\ College of Public Health Sciences, Chulalongkorn University, \\ Phathumwan, Thailand and \\ Center for Risk Analysis and Health Surveillance (C-RAHS), Bangkok, Thailand, and \\ Wattasit Siriwong \\ College of Public Health Sciences, Chulalongkorn University, \\ Phathumwan, Thailand
}

\begin{abstract}
Purpose - The study aimed to evaluate the effectiveness of the Healthy e-Elderly People Intervention (HEPI) mobile application in reducing the physical health effects caused by smartphone usage.

Design/methodology/approach - This randomized controlled trial involved elderly volunteers residing in different regions of Thailand and using smartphones. The samples included 33 participants in each control and intervention group. The intervention group received the HEPI application with reminder messages, while the control group received the HEPI application without reminder messages. Assessments were conducted at baseline, follow-up 1 (four weeks after the last reminder messages) and follow-up 2 (12 weeks after the last reminder messages). Data analyses (i.e descriptive statistics, independent sample $t$-tests and repeatedmeasures analysis of variance) were used to obtain the overall mean change difference between the intervention and control groups at different time points (per-protocol analysis). The priorities of physical health risk were assessed using Health Risk Matrix.

Findings - The HEPI mobile application significantly improved knowledge, attitudes and practice scores in both the HEPI with and without reminder messages. The mean physical health risk score in both control and intervention groups was radically decreased from baseline to follow-up 1; lower physical health scores suggested lower health risk.

Originality/value - Increased duration of smartphone usage by elderly individuals in Thailand may result in a risk of developing several serious health conditions. The HEPI application with reminder messages could be
\end{abstract}

(C) Wachiraporn Wilaiwan and Wattasit Siriwong. Published in Journal of Health Research. Published by Emerald Publishing Limited. This article is published under the Creative Commons Attribution (CC BY 4.0) licence. Anyone may reproduce, distribute, translate and create derivative works of this article (for both commercial and non-commercial purposes), subject to full attribution to the original publication and authors. The full terms of this licence may be seen at http://creativecommons.org/licences/by/4.0/ legalcode

The authors are thankful to the Ratchadapiseksomphot Fund, Chulalongkorn University for the Postdoctoral Fellowship, the grant for International Research Integration: Chula Research Scholar (GCURS_59_06_79_01) Ratchadaphiseksomphot Endowment Fund and the Office of International Affairs Scholarship for Short-term Research, Chulalongkorn University.

Conflict of interests: The author reports no conflicts of interest in this work.

Effectiveness of HEPI mobile application

Received 22 November 2019

Revised 17 June 2020 24 August 2020

Accepted 11 September 2020 
JHR

35,6

used as a tool to benefit smartphone users and would further benefit from a booster after four weeks of intervention.

Keywords Health effects, Smartphone, Elderly, Mobile health, Mobile apps, Thailand

Paper type Research paper

\section{Introduction}

A smartphone is a type of mobile phone that offers more general computing capabilities, supporting a wide range of other services, such as message, Internet access, e-mail and gaming [1]. The number of smartphone users in Thailand has increased from $8.0 \%$ of the Thai population in 2012 to $50.5 \%$ in 2016 [2]. However, the use of smartphones exerts negative effects on physical health, such as headache, dizziness [3], eye pain, musculoskeletal pain, neck pain, shoulder pain and wrist pain [4].

Elderly individuals in Thailand also use smartphones in their daily life. As shown in previous data, the number of elderly mobile phone users was 5,816,966. Of those, 639,911 individuals $(6.4 \%)$ reported using smartphones [5]. The average period of smartphone usage among Thai elderly smartphone users was $3.1 \pm 2.1$ years. The average \pm standard deviation time spent on devices was $2.8 \pm 1.9 \mathrm{~h} /$ day [6]. Previous studies have shown that the most popular application used was reported to be for social networking. Most elderly users had experienced dim eyes, eye pain, shoulder or neck pain, sore muscles and wrist pain $[6,7]$.

One of the factors associated with the effects on physical health among elderly smartphone users was its use for social networking [6], which required the users to hold their arms out in front of them to read. Furthermore, thumb postures while text messaging may lead to pain in the neck and shoulders [8]. Moreover, the too-small size of screens and poor body postures while using smartphones resulted in back pain and neck pain [4,9]. Continuous usage of mobile communication devices is associated with effects on physical health, such as eye pain [6] that may be caused by faster evaporation of the tear film [10]. However, currently, there are no specific intervention programs aimed at reducing the effects of smartphone usage on the physical health of elderly individuals. Therefore, an intervention program for elderly smartphone users should be considered.

The use of smartphones offers certain advantages, such as mobile health (mHealth). The World Health Organization (WHO) defined mHealth as "medical and public health practice supported by mobile devices, such as mobile phones, patient monitoring devices, personal digital assistants, and other wireless devices". The value of mHealth is the production of healthcare practices that become accessible to the community by mobile communication technologies [11]. Furthermore, mHealth becomes a catalyst for observing opportunities in health and promoting healthy behavior for the prevention or reduction of adverse health effects [12].

The Healthy e-Elderly People Assessment (HEPA) application was introduced to perform health risk assessments among smartphone users. The HEPA consists of a 5-part questionnaire including demographic characteristics, usage of smartphones, frequency and magnitude of health effects lasting three months during and after using smartphones based on the participant's perception and judgment, knowledge, attitudes and practices regarding health effects of smartphone use and quality of life [7]. The Healthy e-Elderly People Intervention (HEPI) application was based on the Health Belief Model (HBM) that was developed to examine why people failed to accept protective health measures. It is the most widely active theory of health behavior (Figure 1). The four primary variables consisted of the original HBM which were susceptibility, severity, benefit and barrier. Moreover, from the four primary variables previously mentioned, cue to action determinant was added to the HBM model to represent an activation for health behavior when proper beliefs are supposed. In 1988, self-efficacy was added to the HBM [13]. HEPI was developed to reduce the physical health risk. 


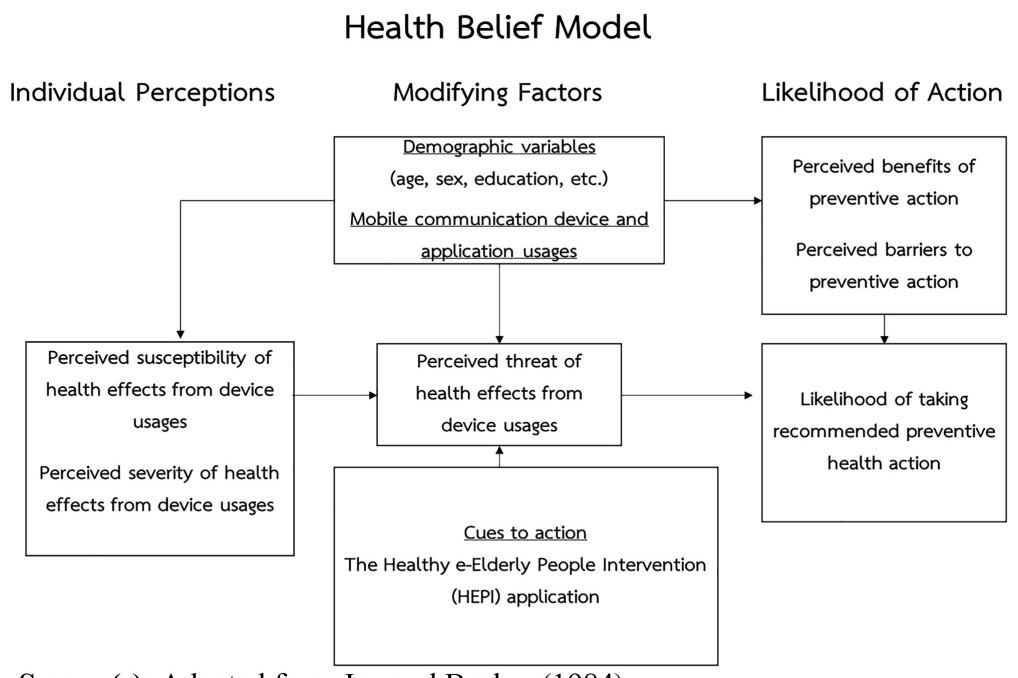

Effectiveness of HEPI mobile application

Source(s): Adapted from Jan and Becker (1984)

Figure 1. Health belief model framework

This study aimed to evaluate the effectiveness of the HEPI mobile application in terms of enhancing knowledge and improving attitudes among smartphone users regarding the health effects related to this activity as well as to promote appropriate practices of smartphone usage. Finally, the focal point was to evaluate the effectiveness of the HEPI mobile application on the physical health effects among elderly smartphone users.

\section{Materials and methods}

This was a randomized controlled trial (single-blinded) involving Thai elderly smartphone users using the mobile application in the same interfaces and smartphone usage as an individual user. The intervention group was provided with the HEPI application and reminder messages, while the control group was provided with the HEPI application without reminder messages. The study area was Thailand. The population was elderly people in Thailand. There were 33 participants in the intervention group, and 33 participants in the control group. This volunteer population included males and females aged 60-80 years, who had experienced moderate or severe physical health effects from smartphone usage, according to an evaluation performed by the Healthy e-Elderly People Assessment (HEPA) mobile application. The physical health risk level came from the frequency and magnitude of health effects lasting three months during and after using smartphones or tablets based on the participant's perception [7]. The sampling technique in this study phase was based on volunteer users of the HEPA application. All volunteers came from all regions of Thailand. Because of the time limitation to this study, after five months of HEPA activity, the recruiting process was stopped. The number of participants recruited was 66 people. The next step involved the randomized allocation of 33 participants into the intervention group and 33 participants into the control group (Figure 2). The study included only users who had an android smartphone and used it for $>6$ months. Individuals who planned to change smartphones were excluded.

\section{Intervention}

The HEPI application was produced within the Thai context and appeared as a free download in the Google Play store for android phones. The intervention group was provided with the 


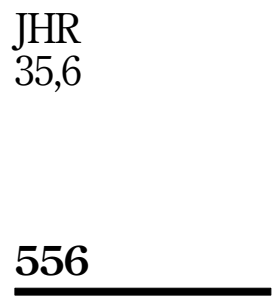

Figure 2.

Flow diagram of recruitment

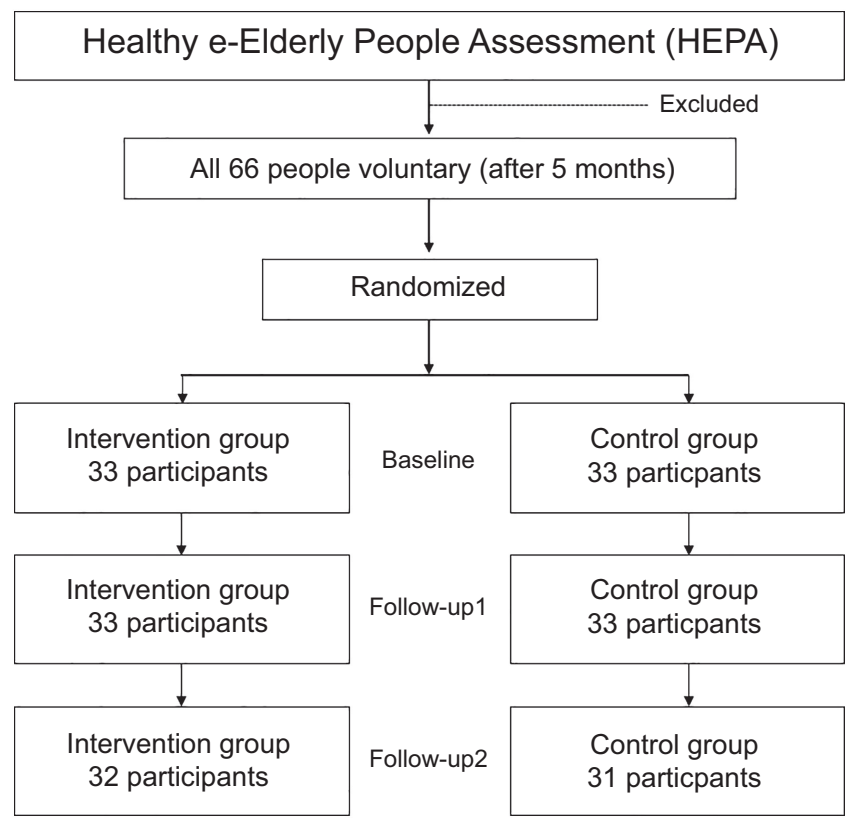

HEPI application and reminder messages, while the control group was provided with the HEPI application without reminder messages. The main page showed four main buttons (i.e. a checklist menu, a notification menu, a "suggestions for practice" menu and a settings menu), in addition to a reminder message function for the intervention group. The checklist menu consisted of seven questions per week, with a running period of four weeks and a total of 28 questions. The checklist was a set of questions requiring "yes" "no" answers such as "Did you turn off your device at least 1-2 $\mathrm{h}$ before sleep last week?". The questions were used to assess the behaviors of elderly users during the previous week and confirm whether they had implemented the practices suggested by the reminder message. The question checklist was related to reminder messages and suggestions for practices. The notification menu showed all the reminder messages that they had received up to that point in time. The "suggestions for practice" menu included information regarding the appropriate use of mobile communication devices to reduce its adverse effects on physical health. This was separated into four groups of symptoms: eye system, nervous system, musculoskeletal system and other systems. Subsequently, each system displayed a suggestion for each symptom via a suggestion line with an image that was related to the suggestion. The setting menu described the details of the project, the researcher and the contact information of the research assistants. Reminder messages appeared every day in the morning and evening which were the time that had the highest number of smartphone users. The reminder message was identical to the suggestions appearing in the practice menu.

\section{Instruments}

The HEPA application is used as a health risk assessment of users. It measured the frequency and magnitude of health effects that occur while using a smartphone or tablet, or after using it for the previous three months. There were 15 effects on physical health (i.e. eye pain, conjunctivitis/dim eyes, red eyes, eye irritation, eye dryness, watery eyes, headache, 
dizziness/nausea, heart palpitations, fatigue/exhaustion, wrist pain, trigger finger, numb finger/hand, shoulder or neck pain/sore muscles, low back pain, sleepless/restless sleep patterns, defecation and urogenital disorders and accidents), 10 effects on mental health and nine effects on social health that were assessed. Knowledge questions about smartphone usage can cause headache, dizziness, red eyes, eye irritation, dry eyes, exhaustion, eye irritation, dry eyes or tear flow, trigger or numb fingers, shoulder or neck pain or sore muscle or low back pain, sleeplessness and bacteria on smartphones. Attitude questions were opinions about smartphone usage and its health effects and opinions about smartphone usage for preventive health effects. Questions relating to practice were asked about their continuing or frequent use of smartphones and practices of smartphone usage while walking on the street, transporting on board, driving and while engaged in activities with the family.

Ethical issue. The experimental protocol was approved by the Ethics Review Committee for Research Involving Human Research Subjects, Health Sciences Group, Chulalongkorn University (COA No. 058/2017; Date of approval: 28 March 2017).

\section{Data collection and analysis}

Research assistants were trained by the researcher, and the researcher gave information to participants at the participant's house or Provincial Public Health Office that participants were living in. Research assistants and researchers installed the HEPI application on the mobile communication device of participants. A questionnaire in the HEPA mobile application had been used for the baseline survey. The assessment that was conducted at baseline and follow-up 1 was collected four weeks after the last reminder messages from the HEPI application and follow-up two was collected 12 weeks after the last reminder messages from the HEPI application. For knowledge regarding the health effects of the communication device and application usage, a correct answer for the knowledge score was given 1 score and 0 scores for wrong answers. The knowledge scores between 8 and 10 were high levels, 6 and 7 were moderate levels, 0 and 5 were low levels. Attitude and practice scores were classified into three levels by using minimum and maximum interval as follows: a score between 13.34 and 20.00 was a good level, 6.67 and 13.34 was a moderate level, and 0 and 6.67 was a poor level score.

The SPSS program version 16 was used to analyze the data. The independent sample $t$-test and Mann-Whitney $U$ test were used to detect differences in continuous variables between the intervention and the control groups at baseline. Repeated measures analysis of variance (ANOVA) was used to detect the overall mean change difference between the groups at different time points (per-protocol analysis).

\section{Results}

The average age in the intervention and control groups was $65.03 \pm 4.77$ and $63.79 \pm 4.34$ years, respectively (Table 1). The comparison between participants in intervention and control groups at baseline among dependent variables found that a significant factor was a total attitude (Table 2).

Following the implementation of the intervention program for four weeks (follow-up 1), the knowledge scores in both the intervention and control groups were increased. At followup 1, the knowledge score in the intervention group (7.90) was higher than that reported in the control group (6.81). After 12 weeks of intervention (follow-up 2), the knowledge scores in both the intervention (5.13) and control (5.56) groups decreased; however, the knowledge scores were higher than those observed at baseline.

The attitude scores of the effects of device usage exhibited a statistically significant difference between the intervention and control groups at baseline. The attitude scores in the
Effectiveness of HEPI mobile application 
JHR 35,6

\section{8}

Table 1.

Comparison of the sociodemographic characteristics between the intervention and control groups at baseline

intervention group were slightly increased from baseline to follow-up 1 (14.35) and slightly decreased at follow-up 2 (13.65). In contrast, the attitude score was significantly increased between baseline and follow-up 2 in the control group. Furthermore, the attitude scores were significantly increased from follow-up 1 (11.22) to follow-up 2 (13.44). Moreover, the attitude in the intervention group was significantly improved versus that recorded in the control group at follow-up 1.

The practice score in the intervention group was markedly increased from baseline to follow-up 1(17.03) and decreased at follow-up 2 (14.90). Nevertheless, the practice score in the control group was slightly increased from baseline to follow-up 1 (13.44) and at follow-up 2 (13.97). The practice score in the intervention group was higher than the practice score reported in the control group at follow-up 1.

The physical health risk score in the intervention group was markedly decreased from baseline to follow-up 1 (5.55) and increased at follow-up 2 (8.35). Similarly, in the control group, the physical health effect risk score was markedly decreased from baseline to followup 1 (7.56) and increased at follow-up 2 (8.88).

Repeated measures ANOVA with adjusted variables (photo application usage and device usage at the workplace) showed the statistically significant difference observed between the intervention and control groups in knowledge regarding the usage of mobile communication devices and applications and the score of the effects of usage at follow-up 1. Besides, there was a statistically significant difference between the intervention and control groups in knowledge at follow-up 2 (Figure 3).

Comparison of the attitude score at different measurement periods did not reveal a statistically significant difference between baseline, follow-up 1 and follow-up 2 in the intervention group. In contrast, there was a statistically significant difference in these scores between baseline and follow-up 2 in the control group $(\phi<0.001)$. Also, there was a

\begin{tabular}{|c|c|c|c|c|c|}
\hline \multirow[b]{2}{*}{ Characteristics } & \multicolumn{2}{|c|}{$\begin{array}{c}\text { Intervention group } \\
\quad(n=33)\end{array}$} & \multicolumn{2}{|c|}{$\begin{array}{c}\text { Control group } \\
(n=33)\end{array}$} & \multirow[b]{2}{*}{$p$-value } \\
\hline & Mean & $\mathrm{SD}$ & Mean & $\mathrm{SD}$ & \\
\hline \multirow[t]{2}{*}{ Age (years) } & 65.03 & 4.77 & 63.79 & 4.34 & 0.273 \\
\hline & $n$ & $\%$ & $n$ & $\%$ & \\
\hline Male & 14 & 42.4 & 16 & 48.5 & 0.621 \\
\hline Married and living together & 27 & 81.8 & 23 & 69.7 & 0.180 \\
\hline Bachelor degree or higher & 16 & 48.5 & 11 & 33.3 & 0.277 \\
\hline Have chronic diseases & 18 & 54.5 & 13 & 39.4 & 0.218 \\
\hline \multicolumn{6}{|c|}{$\begin{array}{l}\text { Note(s): *Significant at } p<0.05 \text {, used Chi-square test } \\
\text { Abbreviation: SD, standard deviation }\end{array}$} \\
\hline
\end{tabular}

Table 2.

Mean scores of knowledge, attitude and practice in smartphones usage by the study group at baseline (independent $T$-test)

\begin{tabular}{lccc}
\hline \multicolumn{4}{c}{ Mean (Standard deviation) } \\
Scores & $\begin{array}{c}\text { Control } \\
n(33)\end{array}$ & $p$-value \\
\hline Knowledge & $3.61(2.62)$ & $3.84(3.24)$ & 0.593 \\
Attitude & $12.77(3.48)$ & $9.25(4.36)$ & $0.002^{*}$ \\
Practice & $13.03(3.20)$ & $12.12(4.04)$ & 0.314 \\
Physical health & $16.94(13.5)$ & $14.31(11.4)$ & 0.407 \\
risk & & &
\end{tabular}

Note(s): *Significant at $p$-value $<0.05$, used independent $T$-test 

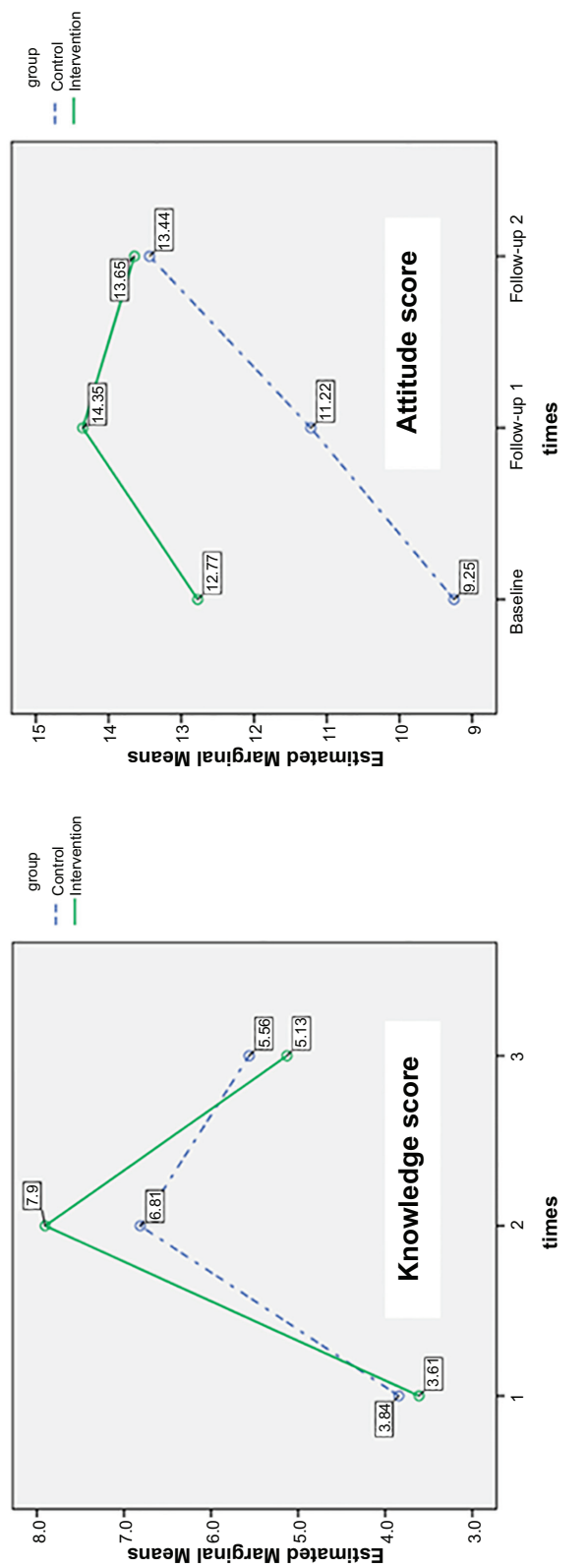

Effectiveness of HEPI mobile application

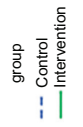

559

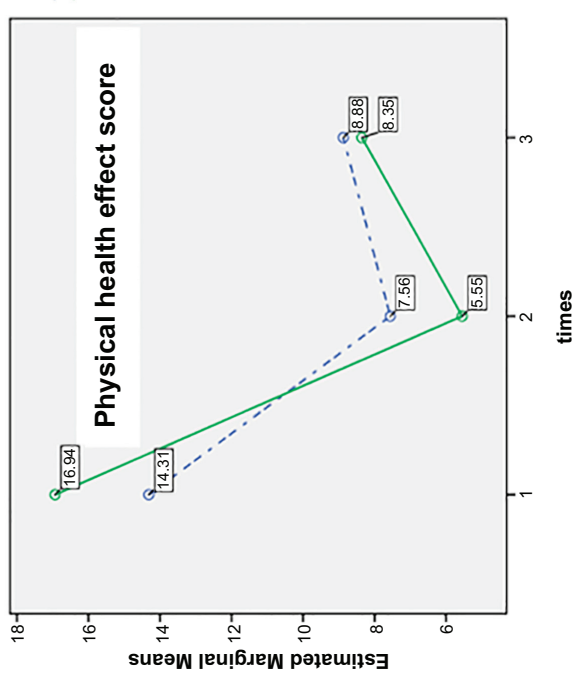

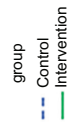

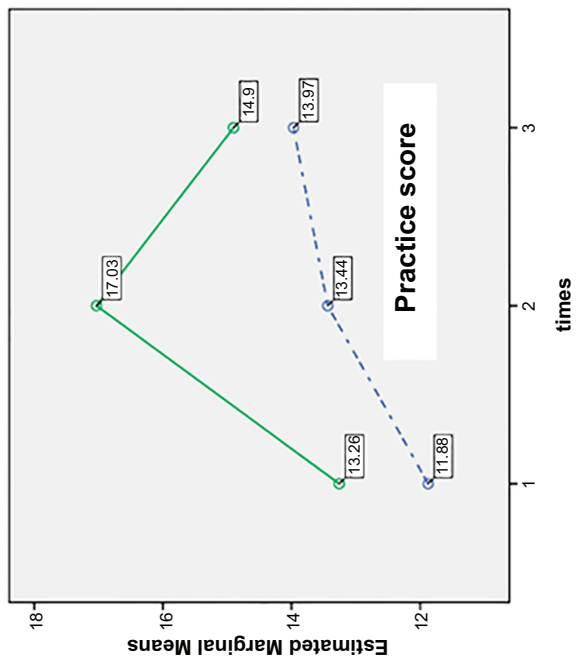

Figure 3.

Change over times of knowledge, attitude, practice and physical health effect scores between intervention and control groups at baseline, follow-up 1 and follow-up 2 
JHR

35,6

statistically significant difference between follow-up 1 and follow-up 2 in this group $(p=0.039)$. At follow-up 1, statistically significant differences were detected between the intervention and control groups in the practice score regarding the usage of mobile communication devices and applications and the score of the effects of usage $(\phi<0.001)$.

There were no statistically significant differences between the intervention and control groups in the physical health effect from mobile communication device usage scores between baseline, follow-up 1 and follow-up 2 .

\section{Discussion}

After the intervention program for four weeks (follow-up 1), the knowledge scores in the intervention group were higher than in the control group at follow-up 1. Both the intervention and control groups used a weekly checklist to report their behavior in terms of device usage during the previous week. Furthermore, both groups had access to recommendations for the appropriate usage of mobile communication devices and applications to reduce the adverse effects on health effects in the "suggestions for practice" menu. Therefore, it may be that the participants in both groups increased their knowledge after installing and using the HEPI application. Similarly, a survey conducted in the USA showed that the majority of healthcare professionals $(86.0 \%)$ believed that the use of health-related applications would increase the knowledge of their patients [14]. In the intervention group, the HEPI application provided daily reminder messages that are similar to SMS texts that were effective in promoting exercise among elderly individuals, but it was not maintained when the SMS text messaging was discontinued [15]. From the result of a previous study, it can be assumed that for the effectiveness of the HEPI application in the intervention group a booster may be required. This finding was similar to those reported in previous studies showing that the Text4baby program increased the knowledge of users regarding prenatal care. Exposure to the text messages of the Text4baby program was associated with changes in beliefs [16].

The increased knowledge score observed in both groups resulted in an increase in the attitude score. The lack of random sampling and the difference of the attitude at baseline might be a reason that the attitude in the control group increased from baseline to follow-up 1 and follow-up 2. Moreover, previous studies showed that knowledge is a structural property of attitudes, showing a link between the number of beliefs and the attitude in memory [17]. The quality of knowledge also influenced the attitude. Moreover, the extent and complexity of knowledge were likely to be positively correlated with the attitude [18]. Three months after discontinuation of the daily reminder messages and weekly checklist, the participants in the intervention group were less motivated, resulting in a slight reduction in the attitude score. The observed increases in the attitude score in the control group from baseline to follow-up 1, and from follow-up 1 to follow-up 2 may be the result of content contamination from other sources. The attitude in the intervention group was significantly improved compared with that reported in the control group at follow-up 1. It is reasonable that individuals in the intervention group receiving daily reminder messages were more motivated. Additionally, the attitude may be affected by the knowledge score [17]. This finding is consistent with those of a previous study showing that the use of a physical activity application in the previous six months resulted in various changes (i.e. attitude, belief, perception and motivation) related to physical activity [19].

In the intervention group, the practice scores were markedly increased from baseline to follow-up 1 and decreased at follow-up 2. A study suggested that knowledge and attitude affect behavior change [20]. Moreover, the HEPI application was based on the Health Belief Model. The participants exhibited perceived susceptibility following the independent health risk assessment performed using the HEPA application. Perceived susceptibility was 
positively associated with improvement in practice. Therefore, the participants had higher practice scores. Moreover, the HEPI may play a role as external cues, respectively, which relate individual performance to the target behavior. Reminders and suggestion strategies may be employed as external assistance in achieving the target behavior [21]. mHealth applications may help the users to self-monitor and motivate themselves to improve their lifestyle in the short and long term [22]. Additionally, mHealth applications have the potential to overwhelm devotion issues by relating with the user with great frequency and as they are executing the behavior. Behavioral change interventions executed through health applications minimize the requirement for face-to-face interactions and thus increase costeffectiveness through pervasive and permanent accessibility [23]. Usage of a physical activity application showed effects of the behavior change theory or mechanisms of change. The results showed an association between self-reported physical activity and behavior [19].

The physical health risk in the intervention group was similar to that of the control group. Of note, lower physical health scores indicated lower health risk. The behavior of the participants improved in both the intervention and control groups. Good practices have been associated with positive health [24]. Previous studies reported that a diabetes management application (WellDoc) provided reminders to users through SMS text messages for monitoring and recording the blood sugar levels of the user. This approach resulted in a significant reduction in the levels of glycated hemoglobin [25]. Another mHealth intervention (MORE Energy) was successful in reducing self-reported fatigue and improving sleep quality and health behavior [26].

A limitation of this study is that symptoms related to the usage of mobile communication devices and applications were self-reported without physical examinations or clinical interviews. In addition, the HEPI application was designed only for Android devices, and this study recruited only elderly users who used Android devices. Short-term follow-up and a lack of no intervention arm might be another limitation. Further studies may assess objective health effects. Also, the intervention should last for a longer time or have a booster.

\section{Conclusions}

The use of the HEPI application with or without reminder messages was effective among the participants. The HEPI application with reminder messages was effective in improving the knowledge, attitude and practice scores, as well as reducing the physical health risks after four weeks of intervention. However, after this point, additional intervention may be required.

\section{References}

1. Northern Lights College. Mobile communication devices (cellular phones/smartphones/mobile internet devices). [updated 2011 Nov; cited 2019 Mar 20]. Available from: http://www.nlc.bc.ca/ Portals/0/documents/policies/a-3_06.pdf.

2. National Statistical Office. Trend of Thais using smartphones. [updated $2017 \mathrm{Nov}$; cited $2019 \mathrm{Mar}$ 20]. Available from: http://www.nso.go.th/sites/2014/Pages/ActivityNSO/A24-05-60.aspx.

3. Shariful Islam SM. Awareness and self-reported health hazards of electromagnetic waves from mobile phone towers in Dhaka, Bangladesh: a pilot study. Adv Public Health. 2014; 2014: 952832. doi: 10.1155/2014/952832.

4. Kim HJ, Kim JS. The relationship between smartphone use and subjective musculoskeletal symptoms and university students. J Phys Ther Sci. 2015; 27(3): 575-79. doi: 10.1589/jpts.27.575.

5. National Statistical Office. The use of computer, internet, and mobile phone. [updated 2016 Nov; cited 2019 Mar 20]. Available from: http://service.nso.go.th/nso/web/statseries/statseries22.html.

6. Wilaiwan W, Rohitrattana J, Taneepanichskul N, Danthamrongkua V, Robson MG, Siriwong W. Health effects of using mobile communication devices: a case study in senior citizens, Thailand. EnvironmentAsia. 2018; 11(2): 80-90. doi: 10.14456/ea.2018.24.
Effectiveness of HEPI mobile application 
JHR

35,6
7. Wilaiwan W, Siriwong W. The Healthy e-Elderly People Assessment (HEPA) application: an evaluation of health effects from smartphone and tablet usage among senior citizens in Thailand. J Health Res. 2019; 33(1): 14-23. doi: 10.1108/JHR-04-2018-0003.

8. Kang JH, Park RY, Lee SJ, Kim JY, Yoon SR, Jung KI. The effect of the forward head posture on postural balance in long time computer based worker. Ann Rehabil Med. 2012; 36(1): 98-104. doi: 10.5535/arm.2012.36.1.98.

9. The Vision Council. DigitEYEzed: the daily impact of digital screens on the eye health of Americans. [updated 2013 Nov; cited 2019 Mar 20]. Available from: https://gunnars.fr/wp-content/ uploads/2014/11/TVCDigitEYEzedReport2013.pdf.

10. Yagci A, Gurdal C. The role and treatment of inflammation in dry eye disease. International Ophthalmology. 2014; 34(6): 1291-1301. doi: 10.1007/s10792-014-9969-x.

11. World Health Organization [WHO]. mHealth: new horizons for health through mobile technologies: second global survey on eHealth. Geneva: WHO; 2011. [cited 2019 Mar 20]. Available from: http:// www.who.int/goe/publications/goe_mhealth_web.pdf.

12. Janz NK, Becker MH. The health belief model: a decade later. Health Education Quarterly. 1984; 11(1): 1-47. doi: 10.1177/109019818401100101.

13. Orji R, Vassileva J, Mandryk R. Towards an effective health interventions design: an extension of the health belief model. Online J Public Health Inform. 2012; 4(3). doi: 10.5210/ojphi.v4i3.4321.

14. Gurol-Urganci I, de Jongh T, Vodopivec-Jamsek V, Atun R, Car J. Mobile phone messaging reminders for attendance at healthcare appointments. Cochrane Database Syst Rev. 2013; (12): CD007458. doi: 10.1002/14651858.CD007458.pub3.

15. Leventhal R. Survey: doctors and patients see benefits in mobile apps. [updated 2015 Nov; cited 2019 Mar 20]. Available from: https://www.healthcare-informatics.com/news-item/survey-doctorsand-patients-see-benefits-mobile-apps.

16. Muller AM, Khoo S, Morris T. Text messaging for exercise promotion in older adults from an upper-middle-income country: randomized controlled trial. J Med Internet Res. 2016; 18(1): e5. doi: 10.2196/jmir.5235.

17. Evans WD, Wallace JL, Snider J. Pilot evaluation of the text4baby mobile health program. BMC Publ. Health. 2012; 12: 1031. doi: 10.1186/1471-2458-12-1031.

18. Krosnick JA, Petty RE. Attitude strength: an overview. In: Petty RE, Krosnick JA, editors. Attitude strength: antecedents and consequences. Mahwah, NJ: Erlbaum Associates; 1995. pp. 1-24.

19. Fabrigar LR, Petty RE, Smith SM, Crites SL, Jr. Understanding knowledge effects on attitudebehavior consistency: the role of relevance, complexity, and amount of knowledge. J Pers Soc Psychol. 2006; 90(4): 556-77. doi: 10.1037/0022-3514.90.4.556.

20. Hoj TH, Covey EL, Jones AC, Haines AC, Hall PC, Crookston BT, et al. How do apps work? An analysis of physical activity app users' perceptions of behavior change mechanisms. JMIR Mhealth Uhealth. 2017; 5(8): e114. doi: 10.2196/mhealth.7206.

21. Schneider B, Cheslock N. Measuring results: gaining insight on behavior change strategies and evaluation methods for environmental education, museum, health, and social marketing programs. San Francisco, CA: Coevolution Institute; 2003. [cited 2019 Mar 20]. Available from: https://www. worldcat.org/title/measuring-results-gaining-insight-on-behavior-change-strategies-andevaluation-methods-from-environmental-education-museum-health-and-social-marketingprograms/oclc/59360114.

22. Holzinger A, Dorner S, Födinger M, Valdez AC, Ziefle M. Chances of increasing youth health awareness through mobile wellness applications. Proceedings of the 6th International Conference on HCI in Work and Learning, Life and Leisure. 2010 November 4-5. Klagenfurt, Austria. Berlin, Heidelberg: Springer; 2010.

23. Melzner J, Heinze J, Fritsch T. Mobile health applications in workplace health promotion: an integrated conceptual adoption framework. Procedia Technology. 2014; 16: 1374-82. doi: 10.1016/j. protcy.2014.10.155. 
24. Belloc NB, Breslow L. Relationship of physical health status and health practices. Prev Med. 1972; 1(3): 409-21. doi: 10.1016/0091-7435(72)90014-x.

25. Quinn CC, Shardell MD, Terrin ML, Barr EA, Ballew SH, Gruber-Baldini AL. Cluster-randomized trial of a mobile phone personalized behavioral intervention for blood glucose control. Diabetes Effectiveness Care. 2011; 34(9): 1934-42. doi: 10.2337/dc11-0366.

26. van Drongelen A, Boot CR, Hlobil H, Twisk JW, Smid T, van der Beek AJ. Evaluation of an mHealth intervention aiming to improve health-related behavior and sleep and reduce fatigue among airline pilots. Scand J Work Environ Health. 2014; 40(6): 557-68. doi: 10.5271/sjweh.3447.

\section{Corresponding author}

Wattasit Siriwong can be contacted at: wattasit.s@chula.ac.th

For instructions on how to order reprints of this article, please visit our website: 